ARTICLE

DOI: $10.1038 / \mathrm{s} 41467-018-06452-2$

\title{
Structural analysis of HTL and D14 proteins reveals the basis for ligand selectivity in Striga
}

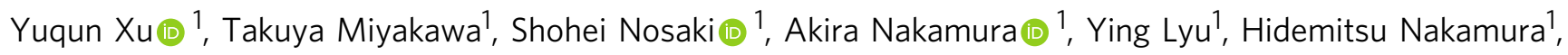
Umeharu Ohto ${ }^{2}$, Hanako Ishida ${ }^{2}$, Toshiyuki Shimizu ${ }^{2}$, Tadao Asami ${ }^{1,3}$ \& Masaru Tanokura (1) ${ }^{1}$

HYPOSENSITIVE TO LIGHT (HTL) and DWARF14 (D14) mediate the perception of karrikin and strigolactone, which stimulates germination of the parasitic weed Striga. However, their role in parasitic seeds is poorly understood, and the basis for their differing responsiveness remains unclear. Here, we show that Striga hermonthica HTL proteins (ShHTLs) in 'conserved' and 'intermediate' clades are able to bind karrikin. The 'divergent' clade is able to hydrolyze strigolactone. Unexpectedly, we find that ShD14 is also capable of hydrolyzing strigolactone. Through comparative analysis of ShHTLs and ShD14 crystal structures, we provide insights into the basis for their selectivity. Moreover, we show that both ShD14 and divergent clade ShHTLs, but not conserved and intermediate clade ShHTLs, can interact with the putative downstream signaling component ShMAX2 in the presence of the synthetic strigolactone, rac-GR24. These findings provide insight into how strigolactone is perceived and how ligand specificity is determined.

\footnotetext{
${ }^{1}$ Department of Applied Biological Chemistry, Graduate School of Agricultural and Life Sciences, The University of Tokyo, 1-1-1 Yayoi, Bunkyo-ku, Tokyo 113-8657, Japan. ${ }^{2}$ Graduate School of Pharmaceutical Sciences, The University of Tokyo, 7-3-1 Hongo, Bunkyo-ku, Tokyo 113-0033, Japan. ${ }^{3}$ Department of Biochemistry, King Abdulaziz University, Jeddah 21589, Saudi Arabia. Correspondence and requests for materials should be addressed to M.T. (email: amtanok@mail.ecc.u-tokyo.ac.jp)
} 
S trigolactones (SLs) and karrikins (KARs) are two classes of structurally related molecules that mediate signaling pathways to stimulate seed germination. SLs induce seed germination of the Striga genus ${ }^{1}$, which are obligate hemiparasites that parasitize the roots of important crops in sub-Saharan Africa $^{2}$, thus causing a reduction in agricultural production by $30-90 \%{ }^{3,4}$. In particular, Striga hermonthica causes more crop loss than any other individual parasite in Africa. In addition to germination stimulation, the root-secreted SLs in the soil also mediate symbiotic associations with arbuscular mycorrhizal fungi ${ }^{5}$, and SLs are also endogenous phytohormones that regulate several aspects of plant growth, such as shoot branching ${ }^{6,7}$. KARs are post-fire germination stimulants found in the smoke of burning vegetation ${ }^{8,9}$ and have been found to regulate seedling development in Arabidopsis ${ }^{10,11}$. Although KARs trigger the germination of numerous plant species, they fail to induce the germination of parasitic weeds ${ }^{12,13}$

SL and KAR have been reported to be recognized by the homologous proteins D14 (DWARF14) ${ }^{14-17}$ and HTL (HYPOSENSITIVE TO LIGHT; also known as KAI2, KARRIKIN INSENSITIVE2 ${ }^{18-20}$, respectively. D14 requires the F-box protein MAX2 (MORE AXILLARY GROWTH2) as the SL signaltransducing component, and MAX2 is also required for KAR signaling ${ }^{21-25}$. It has been reported that D14 is attacked by SL to produce a covalently linked intermediate molecule (CLIM), which induces a conformational change in D14 to facilitate an interaction with MAX2 to trigger SL signaling ${ }^{17}$. By contrast, less is known about KAR signaling. It has been suggested that MAX2 and HTL/KAI2 are involved in KAR signaling based on the experimental observation of the KAR insensitivity of $\max 2$ and kai2-2 mutants ${ }^{18,23}$. The KAR-induced and rac-GR24-induced AtHTL-AtMAX2 interaction has been reported using yeast twohybrid ( $\mathrm{Y} 2 \mathrm{H})$ assays ${ }^{26,27}$.

Although signals of endogenous SL as phytohormones are transduced via the D14-MAX2 system, the perception of exogenous SL in parasite seeds is not well understood. Recently, the perception of SL by S. hermonthica has been reported to be mediated by HTL homologs ${ }^{13,28-30}$. Eleven S. hermonthica HTLs (ShHTLs) have been identified and categorized into three phylogenetic clades: a conserved clade (ShHTL1, also referred to as KAI2c), an intermediate clade (ShHTL2 and ShHTL3, also referred to as KAI2i), and a divergent clade (ShHTL4-11, also referred to as KAI2d) (Supplementary Fig. 1). The conserved clade is under the strongest purifying selection and found in most plants, while the divergent clade is fast evolving and parasite specific. ShHTLs of the different clades showed distinct responses to SL, including natural SLs: strigol, 5-deoxystrigol (5DS), 4deoxyorobanchol and sorgolactone, and a racemic mixture of the synthetic SL analog GR24 (rac-GR24) or KAR in cross-species complementation assays, in which Arabidopsis kai2-2 and htl-3 mutants were transformed with ShHTLs genes: ShHTL1 is not responsive to KAR or SL; ShHTL2 and ShHTL3 are responsive to KAR but not to SL; and ShHTL4-9 are responsive to SL but not $\mathrm{KAR}^{13,29}$. Despite these ShHTLs proteins having a high sequence conservation (sequence identity of more than 60\%), they have distinct SL/KAR specificities and are therefore important for the study of SL/KAR perception. Unveiling the structural basis for the ligand specificities of this series of proteins will help us understand the evolution and discrimination of SL and KAR signals. In contrast, although only one D14 ortholog, ShD14, has been found in Striga, it showed no enzymatic activity toward Yoshimulactone Green (YLG), a fluorogenic SL agonist that becomes fluorescent when it is hydrolyzed by AtD14 or some HTL enzymes ${ }^{27,28}$, despite possessing conserved catalytic triad residues (Supplementary Fig. 2). The molecular mechanism underlying the different responsiveness of these ShHTLs and ShD14 remains vague.
In this study, we evaluate the SL and KAR recognition ability of ShHTLs and ShD14 in vitro and determine the crystal structures of different clades of ShHTLs and ShD14, providing structural insights into the evolution of their SL/KAR selectivity based on a comprehensive structural comparison. Furthermore, interactions between ShHTLs and the putative downstream signaling component ShMAX2 (S. hermonthica MAX2) are determined comprehensively in the present study. These results reveal structural insights into the evolution of ligand specificity of ShHTLs and ShD14 and will provide information for the design of Striga germination stimulants.

\section{Results}

Ligand specificities of ShHTLs and ShD14. The SL and/or KAR responsiveness of ShHTLs has been characterized using transgenic Arabidopsis lines transformed with ShHTL genes in the kai2-2 or $h t l-3$ mutant background ${ }^{13,29}$. However, the signaling machinery is derived from Arabidopsis, and it is still unknown whether the ShHTL homologs bind certain ligands in planta. Based on our previous report ${ }^{31}$ on the ligand specificity of the intermediate clade HTL (ShHTL3, also referred to as ShKAI2i and sharing 98\% identity with ShKAI2iB), we evaluated the ligand specificities of the conserved clade HTL (ShHTL1), the divergent clade HTLs

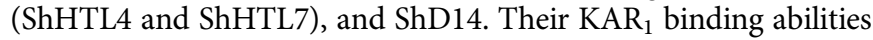
were determined using isothermal titration calorimetry (ITC) assays (Fig. 1a and Supplementary Fig. 3a-d). Only ShHTL1, in the conserved clade, was capable of binding $\mathrm{KAR}_{1}$ (dissociation constant $\left(K_{\mathrm{D}}\right): 77 \pm 12 \mu \mathrm{M}$; means \pm standard deviation $(\mathrm{SD}), n=$ 3) with an affinity comparable to that of ShHTL3 of the intermediate clade $\left(K_{\mathrm{D}}: 78 \pm 3 \mu \mathrm{M}\right)^{31}$. AtHTL is known to be required for KAR responses in Arabidopsis ${ }^{18,23}$. Its binding affinity to $\mathrm{KAR}_{1}$ was also measured using ITC assays in the present study, and the $K_{\mathrm{D}}$ value was determined to be $129 \pm 17 \mu \mathrm{M}$ (Supplementary Fig. 3e), which was comparable to ShHTL1 and ShHTL3. Our results for ShHTL1 binding $\mathrm{KAR}_{1}$ were unexpected because it has been reported that ShHTL1 is not responsive to either $\mathrm{KAR}_{1}$ or rac-GR24 in cross-species complementation assays in terms of seed germination, but rescues some other phenotypes ${ }^{13,32}$ (discussed later). Moreover, no significant $\mathrm{KAR}_{1}$ binding ability of ShHTL4, ShHTL7, and ShD14 was observed in the present study (Fig. 1a; Supplementary Fig. 3b-d).

The SL hydrolytic activity of ShHTLs and ShD14 is important for their function as SL receptors. Therefore, hydrolytic activities toward synthetic SL rac-GR24 and natural SL 5DS were measured by detecting the amount of SL by high-performance liquid chromatography (HPLC) after enzyme treatment (Fig. 1b). ShHTL4 and ShHTL7 were able to hydrolyze both rac-GR24 and 5DS, consistent with a previous report ${ }^{28}$ in which YLG was used to measure their enzymatic activity and their responsiveness to racGR24 and 5DS in cross-species complementary assays ${ }^{13,29}$. Here, we also demonstrated the hydrolytic activity of ShD14 toward racGR24 and 5DS, despite the demonstration of no hydrolytic activity toward YLG in a previous study ${ }^{28}$. To further confirm these results, we repeated the YLG hydrolysis assays using ShD14 and OsD14 and obtained the same results as previously published ${ }^{28}$ (Supplementary Fig. 4). ShD14 was able to hydrolyze rac-GR24 and 5DS but not YLG, suggesting that ShD14 might also serve as an SL receptor. In contrast, ShHTL1 and ShHTL3 showed no hydrolytic activity toward rac-GR24, while ShHTL1 had weak 5DS hydrolytic activity, which was so weak that 5DS-dependent germination was not observed in transgenic lines ${ }^{29}$. Therefore, our results suggested that ShHTL4, ShHTL7, and ShD14 were able to hydrolyze racGR24 and 5DS, but lacked $\mathrm{KAR}_{1}$ binding ability. In contrast, ShHTL1 and ShHTL3 were $\mathrm{KAR}_{1}$ binding proteins without hydrolytic activity toward rac-GR24. 
a
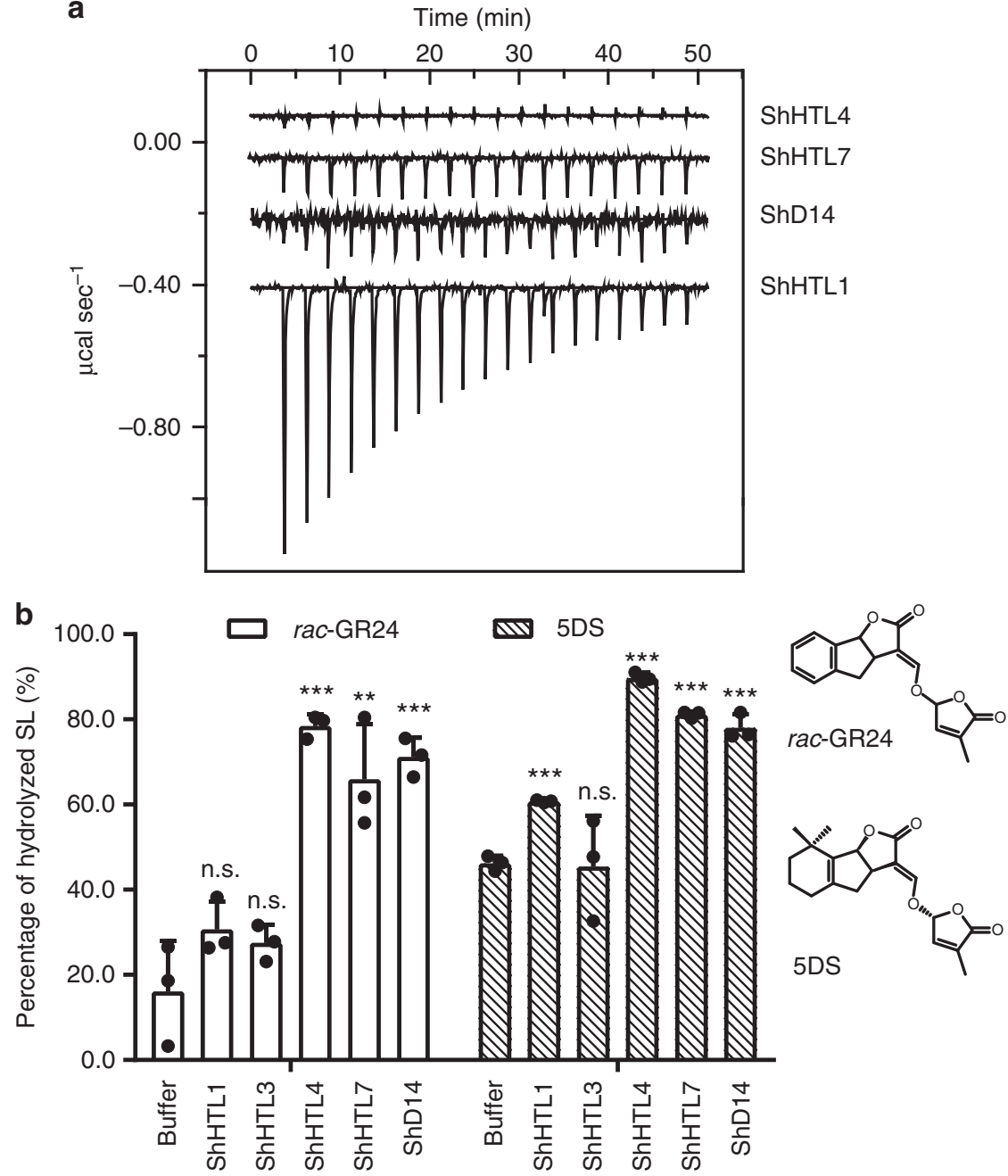

Fig. 1 In vitro ligand specificity of ShHTLs and ShD14. a ITC thermograms titrating KAR $R_{1}$ into ShHTL1, ShHTL4, ShHTL7, and ShD14, respectively. $K_{D}$ of KAR to ShHTL1 was $77 \pm 12 \mu \mathrm{M}$, calculating from three independent experiments (means \pm standard deviation (SD), $n=3$ ). b Hydrolysis of rac-GR24 and 5DS by ShHTLs. rac-GR24 or 5DS was incubated with or without purified enzymes (ShHTL1, ShHTL3, ShHTL4, ShHTL7, and ShD14) for 30 min at $30{ }^{\circ} \mathrm{C}$. HPLC analysis was used to detect the remaining amount of rac-GR24 and 5DS. Columns represent the mean value and error bars the SD of three replicates (means $\pm S D, n=3$ ). Each replicate is indicated by a black dot. The asterisks indicate statistical significance from the buffer (non-enzyme sample) as ${ }^{\star \star *} p \leq 0.001 ;{ }^{\star \star} p \leq 0.01$ and n.s., $p>0.05$, as measured by the one-tailed unpaired $t$ - test

Structural differences in the helix $\mathbf{a D 1}$. To elucidate the structural basis of ligand specificity, we determined the crystal structures of ShHTL1, ShHTL4, ShHTL7, and ShD14 (Fig. 2, Supplementary Fig. 5 and Table 1) and compared their structures with those reported for ShHTL3 ${ }^{31}$ and ShHTL5 ${ }^{29}$. All the structures were highly similar to one another, consisting of a canonical $\alpha / \beta$ hydrolase domain and a helical cap. However, there were apparent structural differences in the helical cap, especially in helices $\alpha \mathrm{D} 1$ (maximum shift of approximately $5 \AA$ ) and $\alpha \mathrm{D} 2$ (Fig. 3a, b). This flexibility of the cap domain is consistent with the observation that helices $\alpha \mathrm{D} 1, \alpha \mathrm{D} 2$, and $\alpha \mathrm{D} 3$ in $\mathrm{D} 14$ underwent a large structural change upon binding to MAX2 ${ }^{17}$. The structural differences in the cap domain appeared to arise from the Y150/F150 residue on loop $\alpha \mathrm{D} 1-\alpha \mathrm{D} 2$ (Fig. 3c). Y150 was changed to F150 in a subgroup (ShHTL4, ShHTL5, and ShHTL7) of the divergent clade, thus losing the hydrogen bond between helices $\alpha \mathrm{D} 1$ and $\alpha \mathrm{D} 3$ (Fig. 3d). Therefore, helices $\alpha \mathrm{D} 1$ and $\alpha \mathrm{D} 2$ of ShHTL4, ShHTL5, and ShHTL7 slid away from $\alpha \mathrm{D} 3$, resulting in larger pockets with a reduced chance of causing steric hindrance with ligands. This finding is interesting because ShHTL4,
ShHTL5, and ShHTL7 have been shown to be highly sensitive to various natural SLs (polyspecific receptors) in a previous study ${ }^{29}$. Therefore, it is speculated that owing to the change in residue 150 , Striga can sense the existence of hosts at very low SL concentrations and recognize various types of SLs that are secreted from hosts.

Structural basis for different ligand specificities. It has been proposed that the pocket size may be the determining factor for recognizing either $\mathrm{KAR}$ or $\mathrm{SL}^{33}$ because KARs are smaller molecules than SLs or GR24. The pocket volume of ShHTLs and ShD14 was estimated to investigate the relationship between the ligand specificity and the pocket size (Fig. 4a). Although similar pocket sizes of ShHTL1 and ShHTL3 have been predicted by modeling, our data showed that the ligand-binding pocket of ShHTL $3^{31}$ was smaller than that of ShHTL1. Such a structural difference between ShHTL1 and ShHTL3 might result in distinct functions, such as the different responses in the germination assays $^{13,29}$. Since structural differences were observed in helices $\alpha \mathrm{D} 1$, and helices $\alpha \mathrm{D} 1$ and $\mathrm{aD} 2$ compose the entrance of the 
ligand-binding pocket, they might contribute to the different sizes of the ligand-binding pockets of ShHTLs and ShD14. It is apparent that the more the helix $\alpha \mathrm{D} 1$ shifts outward (Fig. 3b), the larger the ligand-binding pocket becomes (Fig. 4a). For example, ShHTL7, with the largest pocket, had the helix aD1 tilting

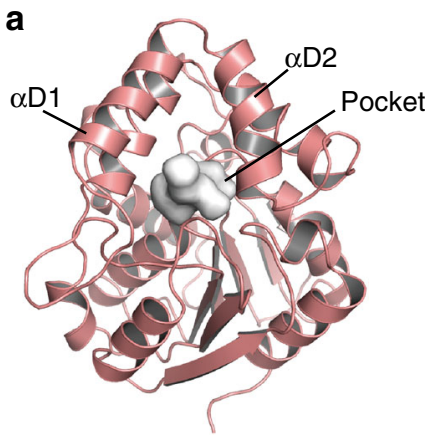

ShHTL1

C

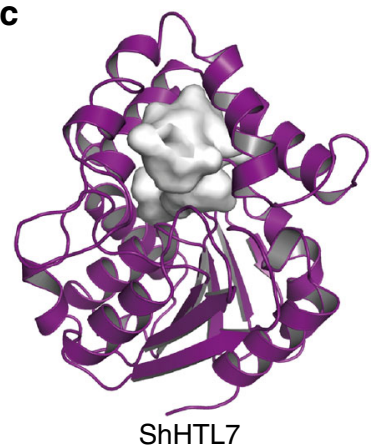

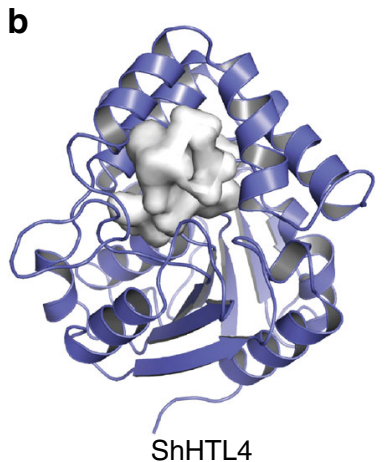

d

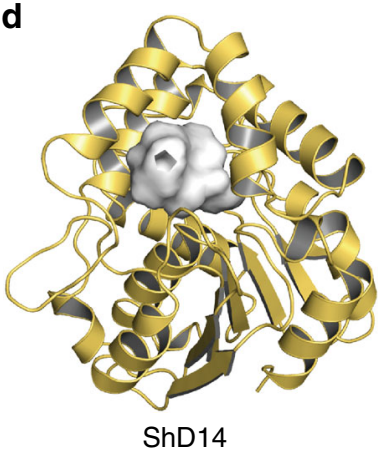

Fig. 2 Overall structures of ShHTLs and ShD14. a-dStructures of ShHTL1 (a), ShHTL4 (b), ShHTL7 (c), and ShD14 (d) are colored pink, slate blue, purple, and yellow, respectively. The ligand-binding pockets are shown in surface representations in white the greatest extent away from the entrance of the pocket. Since the helix $\alpha \mathrm{D} 1$ constituted a part of the entrance to the ligandbinding pocket, it is suggested that the orientation of helix aD1 affected the size of the ligand-binding pockets, and thus the helix $\alpha \mathrm{D} 1$ might be an important structural determinant for the ligand- binding specificity.

ShHTLs of the divergent clade had larger pockets than the D14 group (Fig. 4a). In the divergent clade (ShHTL4, ShHTL5, and ShHTL7), ShHTL7 had the largest pocket. Structural analyses revealed that although the cap domains of ShHTL7 could overlay well with those of ShHTL4 and ShHTL5, with a root-meansquare deviation (RMSD) of $0.9 \AA$ (Supplementary Fig. 6), ShHTL7 had more non-bulky residues than ShHTL4 and ShHTL5, such as T142, L153, and T157 (Fig. 4a, b). It is reasoned that ShHTL7 evolved to use smaller residues to enlarge its pocket while sustaining activity. The larger pocket of ShHTL7 might be related to its high sensitivity to rac-GR24 and its responses to various $\mathrm{SLs}^{28,29}$ due to a reduced chance of steric hindrance upon SL binding. ShD14 and OsD14 have bulky residues in their pockets, such as $\mathrm{F} 125^{\mathrm{ShD} 14} / \mathrm{F} 176 \mathrm{OsD} 14$, $\mathrm{W} 154^{\mathrm{ShD} 14} / \mathrm{W} 205^{\mathrm{OsD} 14}$, and F194 $4^{\mathrm{ShD} 14} / \mathrm{F} 245^{\mathrm{OsD} 14}$ (corresponding to residues 124, 153, and 194 in ShHTLs), making their pockets smaller than those of the divergent clade (Fig. 4a, c). However, these differences between ShD14 and ShHTL7 did not seem to influence their hydrolytic activity toward rac-GR24 (Fig. 1b).

The KAR-binding HTLs (ShHTL1, ShHTL3, and AtHTL) had smaller pockets than the D14 group and ShHTLs of the divergent clade due to more bulky and hydrophobic residues. Especially, residue 190 was substituted by a larger side-chain residue in ShHTLs of the conserved and intermediate clades (L190 ShHTL1/ F190 ShHTL3) (Fig. 4a, d). ShHTL1 and ShHTL3 can bind to KAR but not rac-GR24 (Supplementary Fig. 7) ${ }^{31}$. The ligand-binding pocket of ShHTL1 $\left(321 \AA^{3}\right)$ was composed of the largest number of bulky residues (Fig. 4a), resulting in a smaller pocket than AtHTL $\left(411 \AA^{3}\right)$. The larger pocket size of AtHTL might be the reason why AtHTL exhibited a slightly weaker affinity to $\mathrm{KAR}_{1}$ than ShHTL1 and was able to hydrolyze rac-GR24 to some

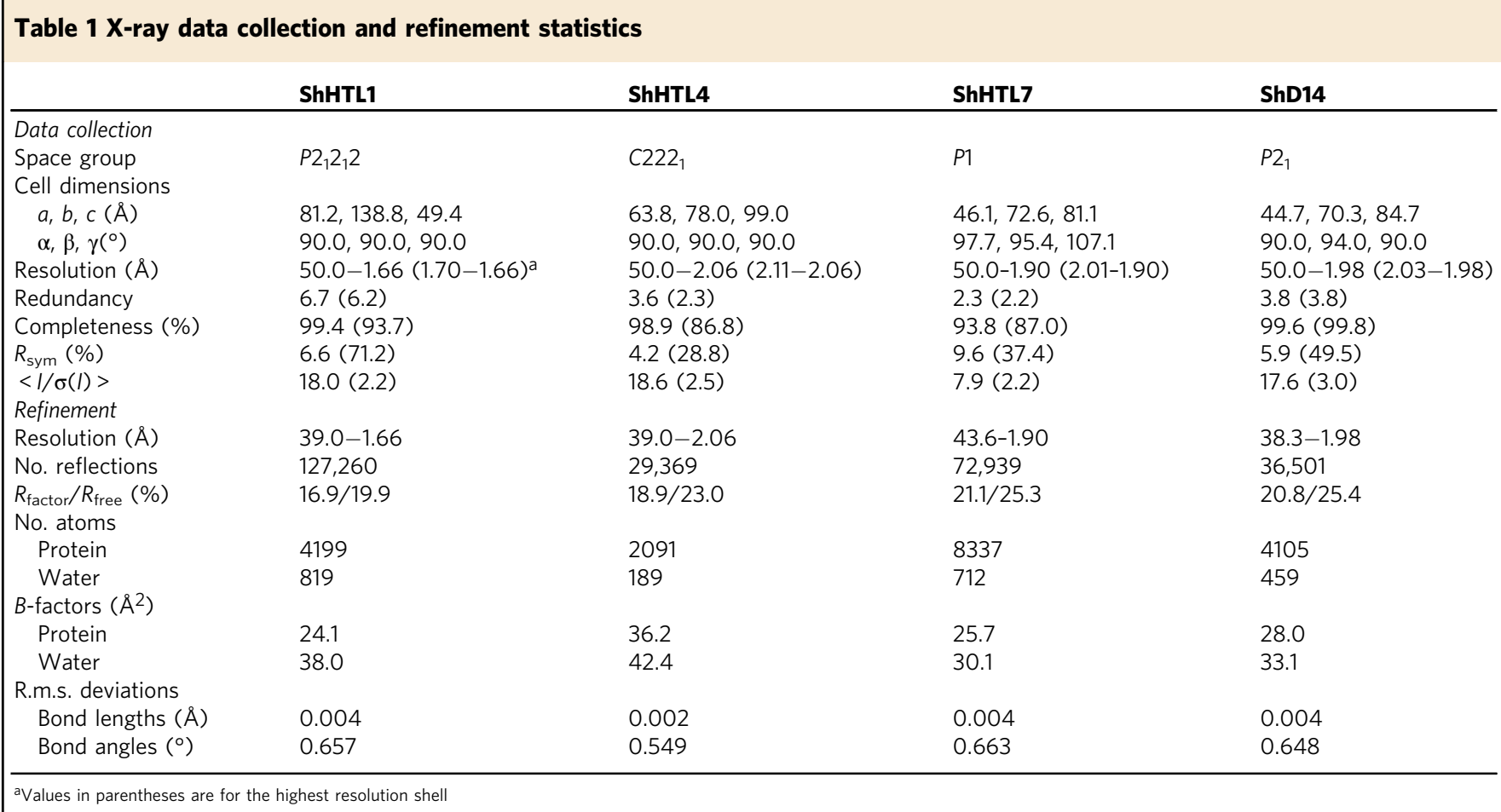


a

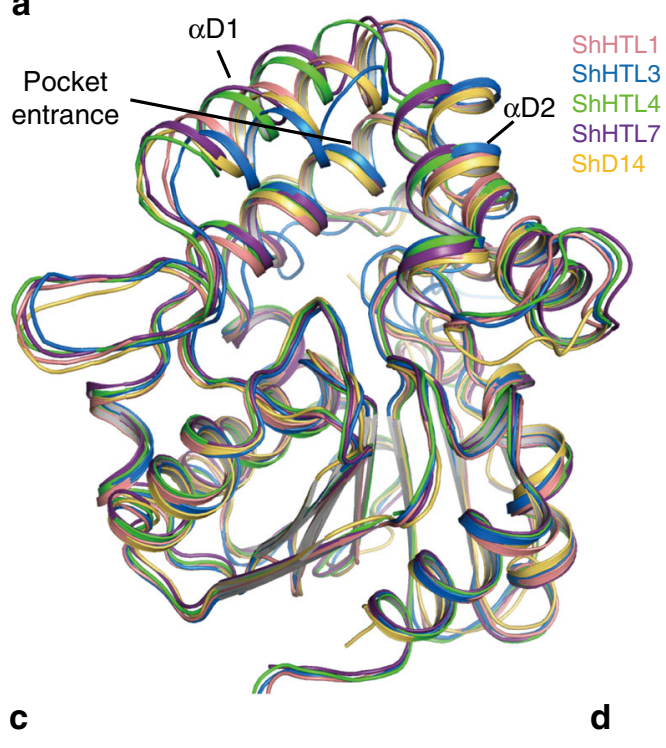

b

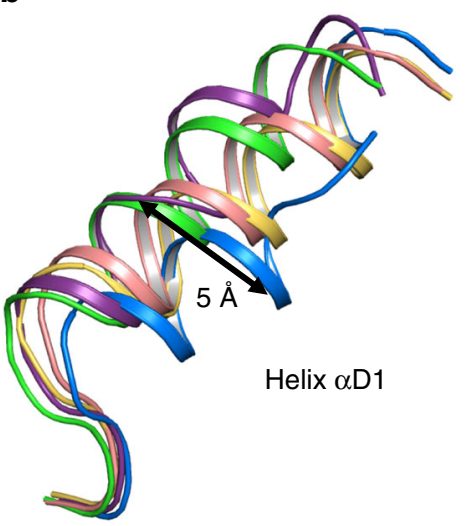

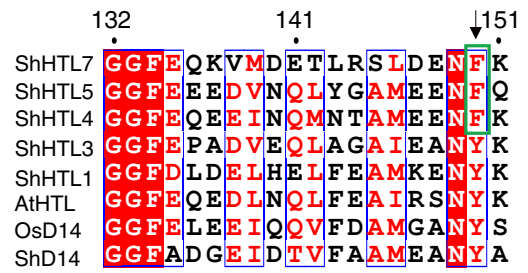

d

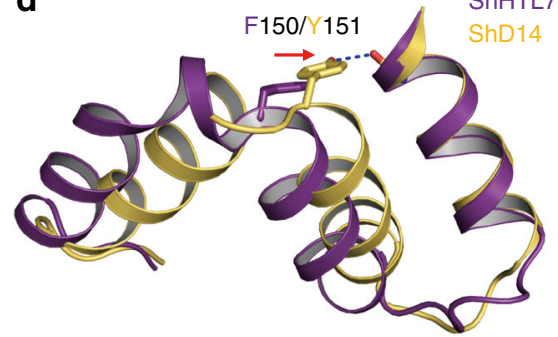

Fig. 3 Structural comparison of ShHTLs and ShD14. a Structural overlay of ShHTL1, ShHTL3 (PDB code: 5DNW [https://doi.org/10.2210/pdb5DNW/ pdb]), ShHTL4, ShHTL7, and ShD14 are colored in pink, blue, green, deep purple, and yellow, respectively. b A detailed view of helix $\alpha$ D1 is shown. The maximum difference between ShHTL3 and ShHTL7 (approx. $5 \AA$ ) is shown by an arrow. c Sequence alignment of residues 132-151 of HTLs and D14s. The green box shows the F150 of ShHTLs in the highly sensitive subgroup (ShHTL4, ShHTL5, and ShHTL7). d Structural alignment of ShHTL7 (deep purple) and ShD14 (yellow) with the hydrogen bond between Y151ShD14 and L178 (main chain carbonyl oxygen atom) is shown as a dashed line

extent $^{26,34}$ and hydrolyze the synthesized desmethyl-YLG (dYLG), which is a smaller version of YLG ${ }^{27}$. Although residues 139 and 143 of ShHTL3 were smaller than those of ShHTL1, ShHTL3 had a shifted helix $\mathrm{aD} 1$ and a closed entrance to the ligand-binding cavity ${ }^{31}$, resulting in the smallest pocket. Due to major bulky residues and the arrangement of the helix $\alpha \mathrm{D} 1$, the pocket sizes of ShHTL1 and ShHTL3 were restricted so that there was only enough space to accommodate KAR.

To verify the importance of pocket size for ligand recognition of ShHTLs, a double mutation T190F/C194F and a triple mutation L124F/T190F/C194F of ShHTL7 were generated to introduce large side-chain amino acids to reduce the pocket size by partially mimicking ShHTL3. Both ShHTL7 mutants were able to bind to $\mathrm{KAR}_{1}$ weakly (Supplementary Fig. 8). The inhibition constant $K_{\mathrm{i}}^{\text {rac-GR24 }}$ in the YLG competition assays was used to indicate the rac-GR24 binding affinity of ShHTL7. It is shown that the T190F/C194F mutation led to a twofold decrease, and the L124F/T190F/C194F mutation led to more than a 100-fold decrease in the rac-GR24 binding affinity (Supplementary Fig. 8). Mutating the residues of the ligand-binding pocket into larger residues reduced rac-GR24 binding and hydrolyzing ability. These results suggested that the bulky residues in the ligandbinding pocket are required to determine the ligand selectivity of ShHTLs.

A structural overlay with the reported GR24 ${ }^{5 D S}$-bound OsD14 structure (PDB code: 5DJ5 [https://doi.org/10.2210/ pdb5DJ5/pdb]) was performed to investigate other determinants of SL selectivity. The structural alignment revealed that GR24 $4^{5 \mathrm{DS}}$ might cause steric hindrance with L142, L/F190, and F194 of the
KAR-binding proteins ShHTL1, ShHTL3, and AtHTL (Supplementary Fig. 9). In contrast, the corresponding residue of L142 in the D14 group was the highly conserved V143 ${ }^{\text {ShD14 }}$ (Supplementary Figs. 9d and 10), which avoids such steric hindrance. Notably, AtHTL hydrolyzes non-natural SLs with a 2'S configuration, such as GR2 $4^{\text {ent-5DS}}$, instead of GR24 $4^{5 \mathrm{DS}}$, which is of natural configuration similar to $5 \mathrm{DS}^{34}$. A previous study has also shown that the non-natural ent-5DS and GR24 $4^{\text {ent-5DS }}$ are able to induce responses similar to SL responses, such as inhibition of hypocotyl elongation and germination of Arabidopsis through AtHTL instead of AtD14 ${ }^{35}$. GR2 $4^{\text {ent-5DS }}$, with an ABC ring in an opposite orientation from GR2 $4^{5 D S}$, likely avoiding the steric clash derived from L142 (Supplementary Fig. 11) and allowing AtHTL to recognize only GR24 $4^{\text {ent-5DS }}$. Moreover, residue 142 of the divergent ShHTLs did not clash with GR24 ${ }^{5 D S}$ due to the outward shift of helix aD1. Similarly, the overlay of the $\mathrm{KAR}_{1}$ bound AtHTL (PDB code: 4JYM https://doi.org/10.2210/ pdb4JYM/pdb) and ShHTL3 (PDB code: 5DNU https://doi.org/ $10.2210 / \mathrm{pdb} 5 \mathrm{DNU} / \mathrm{pdb})$ structures with GR24-binding proteins revealed that residues 157, 218, and/or 219 of the divergent clade ShHTLs and D14 sterically clashed with $\mathrm{KAR}_{1}$ (Supplementary Fig. 12). Therefore, in addition to an appropriate pocket size, residues 142, 157, 218, and 219 are suggested to be determinants of KAR/GR24 selectivity.

Interactions with downstream components. Since the ligand specificity of HTL and D14 is highly related to the signal transduction of these ligands, it is necessary to investigate the 
a

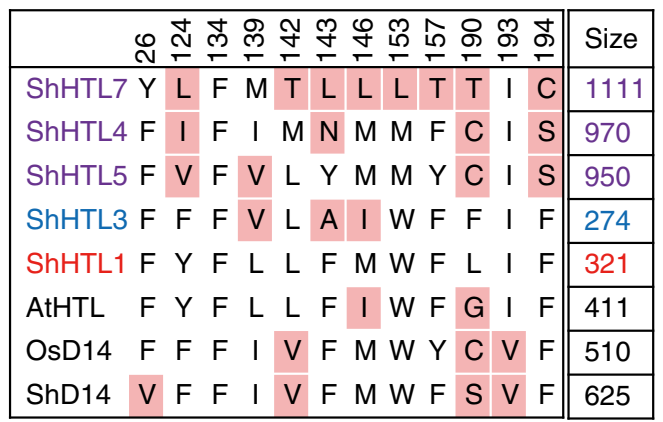

C

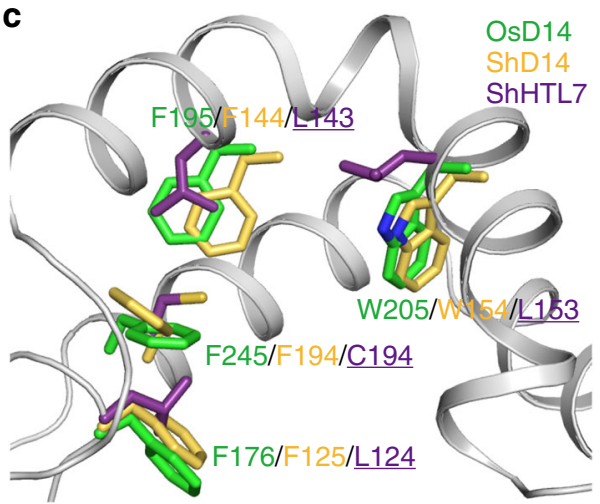

b

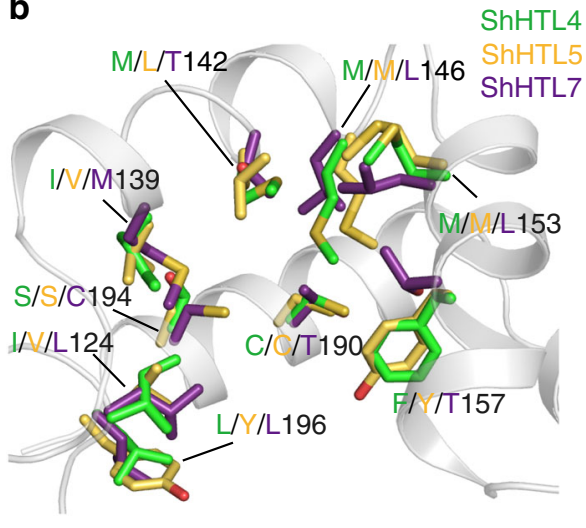

d

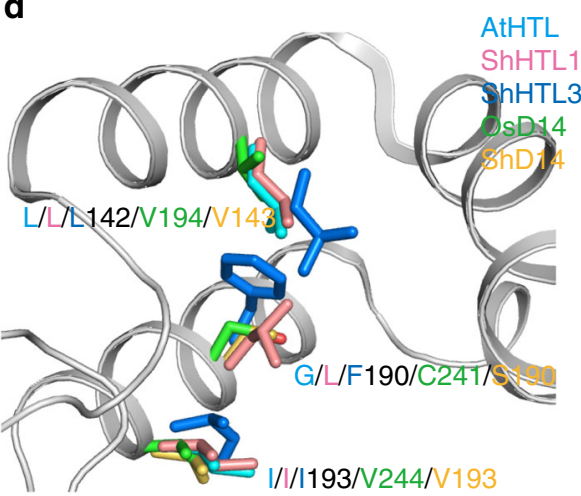

Fig. 4 Residues involved in determining the pocket size of ShHTLs and ShD14. a Residues composing the ligand-binding pocket of each HTL and D14. Font colors of purple, blue, and red represent divergent, intermediate, and conserved clades, respectively, and the background pink color represents less bulky residues. The top row represents the residue number of ShHTL7, and the right column represents the pocket size $\left(\AA^{3}\right)$. b Comparison of residues of ShHTLs of the divergent clade. Residues in the ligand-binding pockets of ShHTL4 (green), ShHTL5 (PDB code: 5CBK [https://doi.org/10.2210/pdb5CBK/ pdb]; yellow), and ShHTL7 (purple) are shown. c Residues composing the ligand-binding pockets of OsD14 (PDB code: 3W04 [https://doi.org/10.2210/ pdb3W04/pdb]; green), ShD14 (yellow), and ShHTL7 (purple) are shown. d Residues composing the ligand-binding pockets of AtHTL (PDB code: 4JYP [https://doi.org/10.2210/pdb4JYP/pdb]; cyan), ShHTL1 (pink), ShHTL3 (blue), OsD14 (PDB code: 3W04 [https://doi.org/10.2210/pdb3W04/pdb]; green), and ShD14 (yellow) are shown

ligand-dependent interactions of ShHTLs and ShD14 with their downstream partners. We first investigated the interactions of ShHTLs and ShD14 with ShMAX2 by using $\mathrm{Y} 2 \mathrm{H}$ assays (Fig. 5a). Our results showed that rac-GR24 triggered the interaction of ShHTL7 with ShMAX2, which was consistent with the results of a previous study ${ }^{30}$. A rac-GR24-dependent ShD14-ShMAX2 interaction was also observed, which strongly suggested that ShD14 might serve as an SL receptor. ShD14 showed a high sequence conservation with other angiosperms D14 (e.g., sequence identity of 75\% with AtD14 and OsD14) and maintained one D14 copy in Striga as in other nonparasitic plants ${ }^{13}$, which strongly suggested that ShD14 had a conserved function with D14 from other angiosperms. In contrast, AtD14 failed to rescue the germination ability of kai22 mutants $^{13}$, suggesting that D14 was not involved in germination. Moreover, the transcripts of ShD14 remained unchanged during the conditioning and germination process of Striga seeds; ${ }^{28}$ therefore, it was likely that ShD14 did not function in germination. Instead, ShD14 might perceive endogenous SL as a phytohormone in Striga, similar to other D14 proteins in nonparasitic plants ${ }^{17}$, which would be consistent with the existence of endogenous SL synthesis components in Striga ${ }^{36}$. Unexpectedly, KAR-binding ShHTL3 failed to interact with ShMAX2 in the presence of KAR ${ }_{1}$. Both of ShHTL1 and AtHTL interacted with ShMAX2 nonspecifically in a ligandindependent manner.
Since $\mathrm{Y} 2 \mathrm{H}$ assays readily detect very weak interactions, in vitro pull-down assays were also performed to further examine the interactions with MAX2. It has been reported that AtMAX2 interacts with AtD14 in a rac-GR24-dependent manner; ${ }^{17}$ therefore, the interaction between AtMAX2 and AtD14 was detected as a positive control (Fig. 5b). Consistent with the results of the $\mathrm{Y} 2 \mathrm{H}$ assays, a direct interaction was detected between ShMAX2 and ShD14 in a rac-GR24-dependent manner, as in the AtD14-AtMAX2 interaction. ShMAX2 was also able to interact with ShHTL4 of the divergent clade in the presence of rac-GR24 (Fig. 5c). No obvious interaction between ShHTL3 and ShMAX2 was detected, but a nonspecific interaction of ShHTL3 with the resin was detected. ShHTL3 was responsive to $\mathrm{KAR}_{1}$ in Arabidopsis ${ }^{13}$ and able to bind to $\mathrm{KAR}_{1}$ in vitro ${ }^{31}$, but the involvement of ShHTL3 and MAX2 in the KAR signaling pathway remains an enigma. Finally, neither the ShHTL1-ShMAX2 (Fig. 5c) nor AtHTL-AtMAX2 (Fig. 5b) interactions were detected. Since weak interactions were detected using $\mathrm{Y} 2 \mathrm{H}$ assays, western blotting was performed to further examine this phenomenon. A weak ligand-independent ShHTL1-ShMAX2 interaction was detected by western blotting, while the AtHTL-AtMAX2 interaction was enhanced by racGR24 (Supplementary Fig. 13a). These interactions were further confirmed using the $\mathrm{Y} 2 \mathrm{H}$ system with a dilution series (Supplementary Fig. 13b, c). A significant difference between the control and the presence of $\mathrm{KAR}_{1}$ or rac-GR24 was not 

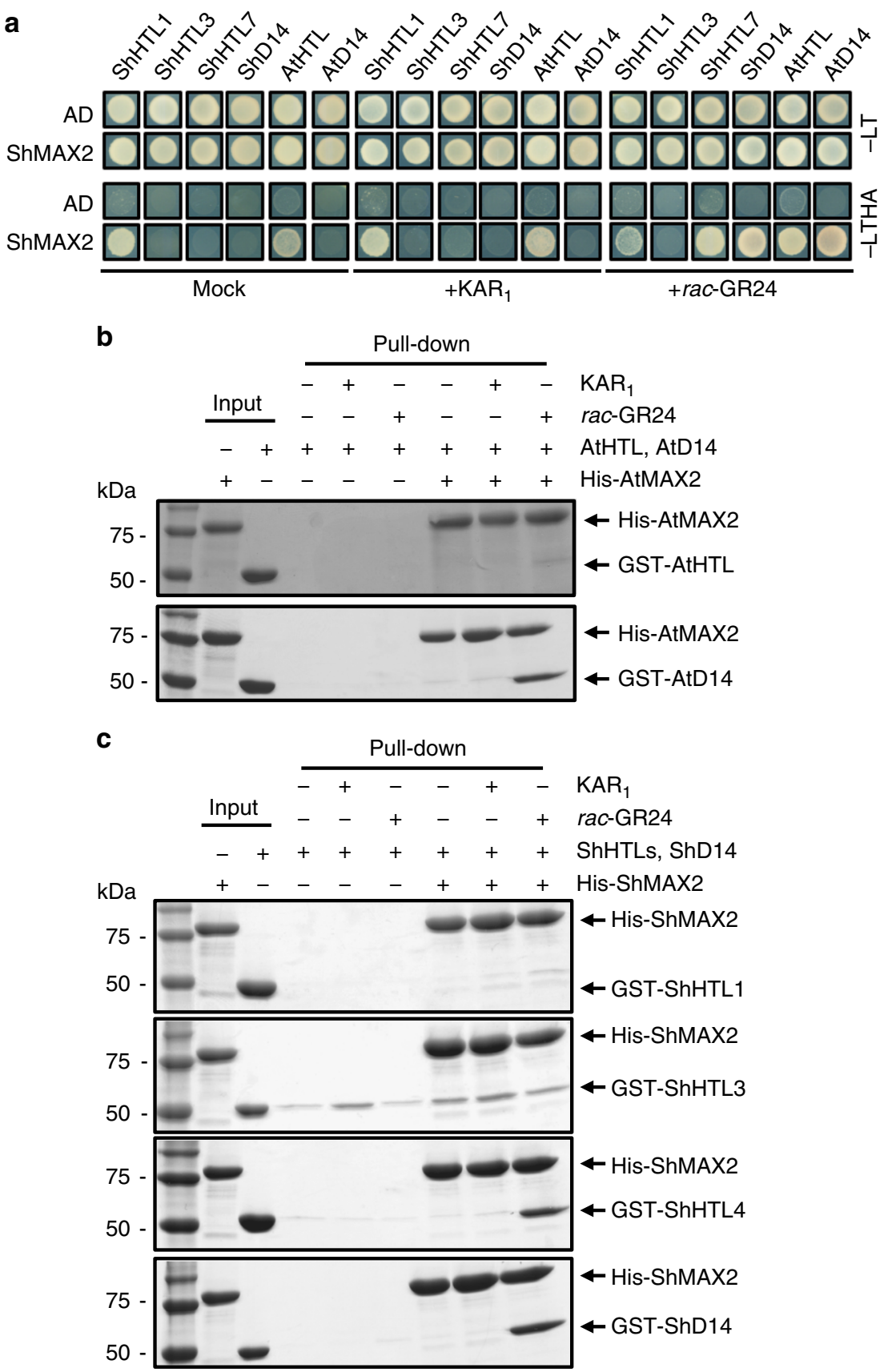

Fig. 5 Interactions between HTLs/D14s and MAX2. a Interactions between HTL/D14 and ShMAX2, as determined by Y2H assays. Yeast cells were cotransformed with binding domain (BD)-fused ShHTL1, ShHTL3, ShHTL7, ShD14, AtHTL, or AtD14 and activation domain (AD)-fused ShMAX2. Interactions between bait and prey were detected in the absence or presence of $5 \mu \mathrm{M} \mathrm{KAR}$ or $5 \mu \mathrm{M}$ rac-GR24 in the control medium (SD/-Leu/-Trp; $-\mathrm{LT}$ ) and the selective medium (SD/-Leu/-Trp/-His/-Ade; - LTHA). b Interactions between AtHTL or AtD14 and AtMAX2 according to the in vitro pull-down assays (detected by Coomassie Brilliant Blue (CBB) staining). His ${ }_{6}$-tag-fused AtMAX2 was used as bait and AtHTL and AtD14 with GST-tags were used as prey. $\mathbf{c}$ Interactions between HTL/D14 and ShMAX2, as determined by the in vitro pull-down assays (detected by CBB staining). In vitro pull-down assays were performed using recombinant $\mathrm{His}_{6}$-tag-fused ShMAX2 as bait. ShHTL1, ShHTL3, ShHTL4, and ShD14 with GST-tags were used as prey. Interactions were tested with or without $50 \mu \mathrm{M} \mathrm{KAR}{ }_{1}$ or $50 \mu \mathrm{M}$ rac-GR24. The full gels are shown in Supplementary Fig. 15

observed for the ShHTL1-ShMAX2 and ShHTL1-AtMAX2 interactions. By contrast, for both AtHTL-AtMAX2 and AtHTL-ShMAX2 cotransformants, better growth was observed in the presence of rac-GR24 than the control and $\mathrm{KAR}_{1}$. These results were consistent with those of the western blotting analysis in the present study and the results of a previous study showing a rac-GR24-induced AtHTL-AtMAX2 interaction in Y2H assays ${ }^{27}$. Therefore, it is suggested that ShHTL1 interacts with ShMAX2 or
AtMAX2 nonspecifically, while the interactions between AtHTL and ShMAX2 or AtMAX2 were induced in the presence of racGR24. Since AtHTL is able to hydrolyze GR24 ent-5DS, becomes thermally unstable in the presence of GR24 $4^{\text {ent }}$-5DS, similar to D14 upon binding to $\mathrm{SL}^{26,34,37}$, and mediates a GR2 $4^{\text {ent }}-5 \mathrm{DS}$ response to regulate plant growth such as inhibition of hypocotyl elongation similar to SL response ${ }^{35}$; it is likely that AtHTL mediates the GR2 $4^{\text {ent }-5 \mathrm{DS}}$ response in a similar manner to the SL 
response mediated by D14. Therefore, the results of our pulldown assays support the hypothesis that to some extent, AtHTL mediates the GR24 $4^{\text {ent }}$-5DS response via the interaction with AtMAX2. Nonetheless, all the examined $\mathrm{KAR}_{1}$-binding proteins failed to directly interact with the MAX2 proteins in a $\mathrm{KAR}_{1}$ dependent manner.

\section{Discussion}

Here, we present the crystal structures of the highly sensitive SL receptor, ShHTL7, as well as ShHTL1, ShHTL4, and ShD14 in Striga. A structural comparison revealed that the arrangement of helix $\alpha \mathrm{D} 1$ and residues in the ligand-binding pocket are two major structural components that determine the pocket size, which in turn determine the KAR or GR24 specificity. The results of this study improve our understanding of the evolution of SL receptors in Striga and provide structural information for the further searches for SL analogs to combat Striga.

The evolutionary model of the different ligand recognition specificities of ShHTLs was improved by the structural findings of the present study (Fig. 6). ShHTL1 of the conserved clade is thought to be the ancestral ShHTL paralog ${ }^{38}$. ShHTL3 of the intermediate clade evolved from the ancestral HTL paralogs and developed the smallest binding pocket due to the presence of P136 and F190, which are proposed as the major structural bases for the ligand specificity of ShHTL $3^{31}$. It is suggested that the divergent clade ShHTLs lost the ability to bind to KAR due to bulky residue 219, and they developed a highly sensitive subgroup (ShHTL4, ShHTL5, and ShHTL7) for SL binding likely via the evolution of F150 and other pocket-composing residues (e.g., residue 194). The divergent clade and intermediate clade are likely to be paralogs that evolved different biological functions during the duplication of the conserved clade.

Although it is well-known that MAX2 is required for both KAR signaling and SL signaling, with the latter requiring the D14-MAX2 interaction to transduce signals, there are few reports investigating the interaction between HTL and MAX2. In the present study, the interactions between ShHTLs and ShMAX2 were comprehensively determined. ShHTL4-ShMAX2, ShHTL7-ShMAX2, and ShD14-ShMAX2 interactions were induced in a rac-GR24-dependent manner. Weak AtHTL-AtMAX2 interactions were detected in the presence of rac-GR24. In contrast, AtHTL unexpectedly did not interact with AtMAX2 in a $\mathrm{KAR}_{1}$-dependent manner. Consistent with this observation, other $\mathrm{KAR}_{1}$-binding HTLs in Striga, ShHTL1, and ShHTL3, showed no direct interaction with ShMAX2 in the

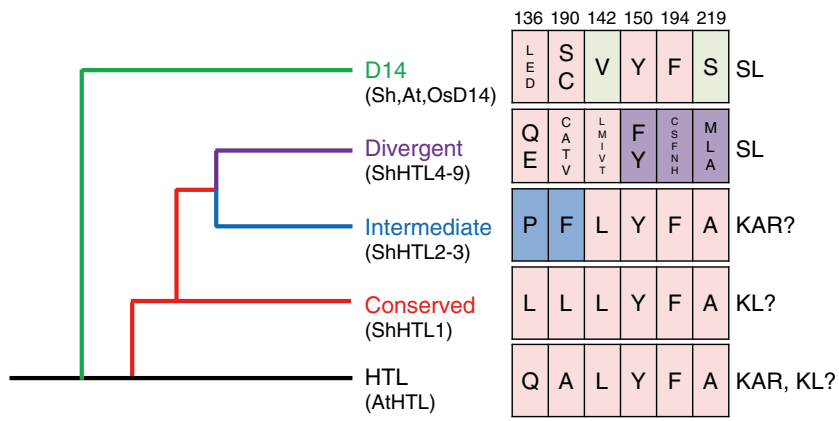

Fig. 6 Model of the evolution of HTL/D14 proteins with different ligand specificities. The evolutionary model of ShHTLs was generated according to a previous model ${ }^{13}$ with modifications (left). ShHTLs of the conserved, intermediate, and divergent clades are colored red, blue, and purple, respectively. The D14 clade is colored green. The residues determining their ligand specificities and the corresponding ligands are shown on the right. The residue number of ShHTL7 is shown on the top presence of $\mathrm{KAR}_{1}$, and the interaction between AtHTL and AtMAX2 has been questioned ${ }^{27}$. It is possible that MAX2 is involved in KAR signaling without any interaction with HTL or that a third component is required to enhance the HTL-MAX2 interaction, indicating a different pathway from SL signaling (Supplementary Fig. 14). Since DWARF53 (D53; a repressor of SL signaling) is able to interact with MAX2 and D14 ${ }^{39,40}$, members of the D53 family or its homologous SUPPRESSOR OF MAX2 1 LIKE (SMXL) family ${ }^{41-44}$ (SMAX1; SMXL6/7/8) might be involved in enhancing the HTL-MAX2 interaction by forming a ternary complex. Alternatively, $\mathrm{KAR}_{1}$ might fail to induce conformational changes in HTL similar to those observed in D14 in the presence of rac-GR24 and MAX2 ${ }^{17}$. This speculation is supported by the evidence that the residues involved in the interaction with MAX2 are conserved between GR24-binding HTLs and the $\mathrm{KAR}_{1}$-binding HTLs (Supplementary Fig. 2). In this case, $\mathrm{KAR}_{1}$ might need to be converted into an active form in vivo for signal transduction to occur (Supplementary Fig. 14).

Our observation of $\mathrm{KAR}_{1}$ binding to ShHTL1 is unexpected because previous studies have shown that ShHTL1 is nonresponsive to exogenous $\mathrm{KAR}_{1}$ in terms of Arabidopsis germination, and that $\mathrm{KAR}_{1}$ fails to induce germination of Striga ${ }^{12,13}$. Although the $K_{\mathrm{D}}$ value for KAR binding of ShHTL1 $(77 \mu \mathrm{M})$ was higher than the physiological concentration $(1 \mu \mathrm{M})$, this result might be because the fraction of active protein in the sample was lower than $100 \%$, resulting in lower estimated affinity than real affinity. Moreover, similar results have been observed for some abscisic acid (ABA) receptors. For instance, PYRABACTIN RESISTANCE 1 (PYR1) has a $K_{\mathrm{D}}$ for $\mathrm{ABA}$ of $97 \mu \mathrm{M}^{45}$. Surprisingly, when PYR1 forms a complex with the co-receptor TYPE 2C PROTEIN PHOSPHATASE (PP2C), the binding affinities for $\mathrm{ABA}$ are in the nanomolar range. Thus, ShHTL1 might need other proteins or other factors to enhance the KAR binding affinity. It is also possible that ShHTL1 is involved in other signaling pathways instead of KAR-induced germination. KAR is a class of abiotic chemical derived from smoke, but kai2-2 mutants and max2 mutants in Arabidopsis both exhibit phenotypes of a reduced photomorphogenic response and increased primary dormancy, indicating that HTL and MAX2 are likely to be involved in the regulation of unknown endogenous phytohormones (termed KL or KAI2 ligand) $23,32,46$. Therefore, it is believed that AtHTL is involved in both KAR signaling and endogenous KL signaling $23,32,46$. Rather than the KAR receptor, ShHTL1 is likely to be the receptor for KL, an endogenous signaling molecule that shares structural similarity with KAR, in particular the butenolide ring. Indeed, conserved clade HTL orthologs exist in most Lamiids as a basal clade, implying their essential roles (e.g., KL pathway) in plants. Therefore, to fully understand the functions of HTL, further studies will be required to investigate the mysterious KL.

\footnotetext{
Methods

Overexpression and purification of recombinant proteins. The coding regions of ShD14 (GenBank: KR013120.1), ShHTL1 (GenBank: KR013121.1), ShHTL4 (GenBank: KR013124.1), and ShHTL7 (GenBank: KR013127.1) were amplified by reverse transcription PCR, using the total RNA of S. hermonthica seeds. Recombinant ShHTL3 was produced as previously described ${ }^{31}$. ShD14 was cloned into the expression vector pET-49b (+) (Novagen). ShHTL1 and ShHTL4 were cloned into pGEX-6P-3 (GE Healthcare) with an N-terminal glutathione S-transferase (GST)tag and a PreScission protease cleavage site. ShHTL7 was cloned into pET-28a (+) (Novagen) between the restriction enzyme sites NcoI and XhoI with a C-terminal His-tag. The recombinant proteins were expressed in the E. coli strain Rosetta (DE3) (Novagen). Cells were harvested by centrifugation at $5000 \mathrm{rpm}$ for $10 \mathrm{~min}$ and stored at $-80^{\circ} \mathrm{C}$ until use. The harvested cells were resuspended in buffer $\mathrm{A}$ (20 mM Tris- $\mathrm{HCl}, \mathrm{pH} 8.0,300 \mathrm{mM} \mathrm{NaCl}$ and $1 \mathrm{mM} \mathrm{DTT}$ ) for GST-tagged proteins or in buffer B (20 mM Tris- $\mathrm{HCl}, \mathrm{pH} 8.0,300 \mathrm{mM} \mathrm{NaCl}, 5 \%$ glycerol and $10 \mathrm{mM}$ imidazole) for His-tagged ShHTL7, and they were lysed by sonication. The cell debris was removed by centrifugation at $40,000 \times g$ for $30 \mathrm{~min}$. The supernatant fractions of ShD14, ShHTL1, and ShHTL4 were then applied to Glutathione
} 
Sepharose $4 \mathrm{~B}$ resin (GE Healthcare). After washing with buffer A, the protease HRV3C was added to remove the GST-tag, and the untagged proteins were then eluted with buffer $\mathrm{A}$. The eluate was further purified by loading onto a Resource $\mathrm{Q}$ or a Mono Q column (GE Healthcare), and elution was performed with a linear gradient of 0-1.0 M NaCl. ShHTL1 was further applied to a HiLoad 26/60 Superdex $75 \mathrm{pg}$ column (GE Healthcare) to remove protein impurities. The supernatant fraction of ShHTL7 was applied to the Ni-NTA superflow resin (Qiagen), washed with buffer containing $20 \mathrm{mM}$ imidazole, and eluted with buffer containing $200 \mathrm{mM}$ imidazole. The eluate was then loaded onto a Mono $\mathrm{Q}$ column (GE Healthcare). All purified proteins were concentrated with Vivaspin 20 devices (10,000 MWCO PES) (Sartorius).

The DNA encoding ShMAX2 (GenBank: JX565467.1), AtMAX2 (GenBank: AF305597.1), and ASK1 (GenBank: AF059294.1) were cloned into the pFastBac Dual vector (Thermo Fisher Scientific) between the restriction enzyme sites EcoRI and BamHI. All the cloned genes were fused to an $\mathrm{N}$-terminal $\mathrm{His}_{6}$-tag following a FLAG-tag and PreScission protease cleavage site. Spodoptera frugiperda 9 (Sf9) insect cells were cultured in Sf-900 II SFM medium containing 50 units $\mathrm{mL}^{-1}$ penicillin, $50 \mu \mathrm{g} \mathrm{mL}^{-1}$ streptomycin, and $5 \%$ fetal bovine serum (FBS). For coexpression of MAX2 with ASK1, the baculovirus encoding ShMAX2 or AtMAX2 was co-infected in Sf9 insect cells with the baculovirus encoding ASK1. The cells were harvested after 3 days of incubation by centrifugation at $3000 \mathrm{rpm}$ for $8 \mathrm{~min}$. The harvested cells were resuspended in $20 \mathrm{mM}$ Tris- $\mathrm{HCl}, \mathrm{pH} 7.4,300 \mathrm{mM} \mathrm{NaCl}$, $10 \%$ glycerol, $1 \mathrm{mM}$ DTT, and $10 \mathrm{mM}$ imidazole and lysed by sonication. The cell debris was removed by centrifugation at $40,000 \times g$ for $30 \mathrm{~min}$. The protein was purified by Ni-NTA superflow resin, followed by a HiLoad 26/600 Superdex $200 \mathrm{pg}$ column (GE Healthcare).

Isothermal titration calorimetry. The binding assays were performed using a MicroCal iTC ${ }_{200}$ isothermal titration calorimeter (GE Healthcare). Prior to the ITC experiments, concentrated ShD14, ShHTL1, ShHTL4, and ShHTL7 were exchanged into a buffer consisting of $20 \mathrm{mM}$ HEPES (pH 8.0) and $150 \mathrm{mM} \mathrm{NaCl}$ to remove dithiothreitol and were then adjusted to a final concentration of $150 \mu \mathrm{M}$. The sample cell was filled with protein solution $(204 \mu \mathrm{L})$. Nineteen consecutive 2.0$\mu \mathrm{L}$ aliquots of $3 \mathrm{mM} \mathrm{KAR}{ }_{1}$ (3-methyl-2H-furo [2,3-c] pyran-2-one) were injected into a prepared protein solution at $150 \mathrm{~s}$ intervals at $20^{\circ} \mathrm{C}$. The first injection volume was $0.4 \mu \mathrm{L}$, and the observed thermal peak was excluded from the data analysis. A negative control was obtained by titrating $3 \mathrm{mM} \mathrm{KAR}_{1}$ into the buffer (20 mM HEPES, pH 8.0, $150 \mathrm{mM} \mathrm{NaCl}$ ) in the same manner. Data fitting was performed in "one set of sites" mode, and $K_{\mathrm{D}}$ values were calculated using Origin software provided by MicroCal.

YLG hydrolysis assays. The YLG assays were conducted as previously described ${ }^{28}$ with minor modifications. Ten microliters of $0.1 \mathrm{mg} \mathrm{mL}^{-1}$ ShHTL7 protein in a reaction buffer containing $20 \mathrm{mM}$ HEPES, pH $7.0,150 \mathrm{mM} \mathrm{NaCl}$ and $80 \mu \mathrm{L}$ of reaction buffer were mixed in a 96-well black plate (Grenier). Ten microliters of $10 \mu \mathrm{M}$ YLG was added to the solution and incubated at $25^{\circ} \mathrm{C}$ for $1 \mathrm{~h}$ prior to measurement. The fluorescence intensity was measured using a SpectraMax iD5 Multi-Mode Microplate Reader (Molecular Devices). Measurements were performed at room temperature under a $480 \mathrm{~nm}$ excitation wavelength, a $530 \mathrm{~nm}$ emission wavelength, a manual PMT of 500, and a read height of $1.00 \mathrm{~mm}$. The fluorescence can be detected if YLG is hydrolyzed. The RFU (relative fluorescence unit) was calculated by subtracting the fluorescence of the buffer (without protein) and then dividing by the fluorescence of wild-type ShHTL7. The experiment was repeated 6 times independently.

HPLC. For the SL hydrolysis assays, $0.5 \mathrm{~mL}$ of PBS buffer (pH 7.3) containing $3.2 \mu \mathrm{M}$ rac-GR24 (racemic mixture of a GR2 $4^{5 \mathrm{DS}}$ and GR2 $2^{\text {ent }}$-5DS ) or 5DS was mixed separately with $1.6 \mu \mathrm{M}$ purified ShHTLs and ShD14 and incubated for $30 \mathrm{~min}$ at $30^{\circ} \mathrm{C}$. Next, $50 \mu \mathrm{L}$ of $1 \mathrm{M} \mathrm{HCl}$ was added to each reaction solution, and the reaction solutions were extracted 3 times with $300 \mu \mathrm{L}$ of ethyl acetate. The ethyl acetate layers were combined and dried in vacuo and were then dissolved in $50 \mu \mathrm{L}$ of methanol. For each layer, $5 \mu \mathrm{L}$ was applied to the reverse-phase HPLC (Shimadzu LC-2030C). The analytical column was a CAPCELL CORE C18 (Ф $2.1 \times$ $100 \mathrm{~mm}$, Shiseido). The analytes were eluted under gradient conditions at a flow rate of $0.20 \mathrm{~mL} \mathrm{~min}^{-1}$ with a linear ramp of methanol to $90 \%$ methanol at $19 \mathrm{~min}$, which was maintained for 6 min before resetting to the original conditions. The amount of rac-GR24 or 5DS was calculated by the peak area (detection wavelength: $254 \mathrm{~nm}$ ) at retention times ranging from 12.2-12.4 min with the regression equation obtained from the calibration curve produced using a dilution series of rac-GR24 or 5DS solutions. The percentage of hydrolyzed SL was calculated and used for statistical analyses. One-tailed unpaired Student's $t$ - tests were used for comparison between buffer samples and enzyme samples.

Yeast two-hybrid (Y2H) assay. Full-length sequences of ShD14, ShHTL1, ShHTL3, ShHTL4, ShHTL7, and AtHTL were cloned into the pGBKT7 vector as bait (Clontech), and the coding regions of ShMAX2 and AtMAX2 were cloned into the pGADT7 vector as prey (Clontech). MAX2 was supposed to be unstable without ASK1. Therefore, AtASK1 was cloned into the N terminus of ShMAX2 and AtMAX2 with a 16-residue linker for co-expression. The bait and prey plasmids were co-transformed into the yeast (Saccharomyces cerevisiae) strain Y2HGold (Clontech) by the lithium acetate-mediated method. The presence of both plasmids was confirmed by growth on SD/-Leu/-Trp (-LT) plates. The interactions between bait and prey in the control medium (-LT) and selective medium (SD/-Leu/ -Trp/-His/-Ade; -LTHA) in the absence or presence of $5 \mu \mathrm{M} \mathrm{rac-GR24}$ (racemic mixtures) or $5 \mu \mathrm{M} \mathrm{KAR}$ were detected. The plates were incubated for 5 days at $30^{\circ} \mathrm{C}$

In vitro pull-down assays. Ni-NTA pull-down assays were performed with purified ShMAX2 or AtMAX2 as the bait and GST-ShHTLs, GST-ShD14, or GSTAtD14 as the prey. The molecular weight of the HTL/D14 proteins is nearly the same as that of ASK1; therefore, the HTL/D14 proteins were fused to GST, increasing their molecular weight for detection on SDS-PAGE gels. For this purpose, $25 \mu \mathrm{g}$ of ShMAX2-ASK1 was mixed with $10 \mu \mathrm{g}$ of GST-ShHTL1, GSTShHTL3, GST-ShHTL4, or GST-ShD14 in the presence of $50 \mu \mathrm{M} \mathrm{KAR}_{1}, 50 \mu \mathrm{M} \mathrm{rac}$ GR24, or a corresponding concentration of DMSO. After incubation at $4{ }^{\circ} \mathrm{C}$ for $30 \mathrm{~min}$, the reaction mixtures were added to $100 \mu \mathrm{L}$ of $50 \%(\mathrm{v} / \mathrm{v})$ Ni-NTA superflow resin slurry that was pre-equilibrated with buffer containing $50 \mathrm{mM}$ Bis-Tris, $\mathrm{pH} 6.8,100 \mathrm{mM} \mathrm{NaCl}, 25 \mathrm{mM}$ imidazole, and $10 \%$ glycerol. After further incubation at $4{ }^{\circ} \mathrm{C}$ for $30 \mathrm{~min}$, unbound proteins were washed with buffer containing $50 \mathrm{mM}$ Bis-Tris, $\mathrm{pH} 6.8,100 \mathrm{mM} \mathrm{NaCl}, 50 \mathrm{mM}$ imidazole, and $10 \%$ glycerol. The protein complexes were then eluted with $100 \mu \mathrm{L}$ of elution buffer containing $50 \mathrm{mM}$ Bis-Tris, $\mathrm{pH} 6.8,500 \mathrm{mM} \mathrm{NaCl}$, and $500 \mathrm{mM}$ imidazole and subjected to SDS-PAGE analysis.

Samples of GST-ShHTL1 and GST-AtHTL were also subjected to western blotting after separation by SDS-PAGE. The proteins were transferred from the SDS-PAGE gel to a polyvinylidene fluoride (PVDF) membrane (Merck Millipore). The membrane was blocked by incubation in TBST buffer $(50 \mathrm{mM}$ Tris- $\mathrm{HCl}, \mathrm{pH}$ $7.4,138 \mathrm{mM} \mathrm{NaCl}, 2.7 \mathrm{mM} \mathrm{KCl}$, and $0.1 \%(\mathrm{v} / \mathrm{v})$ Tween-20) with the addition of $5 \%$ $(\mathrm{w} / \mathrm{v})$ bovine serum albumin for $1.5 \mathrm{~h}$. Next, the membrane was incubated with anti-GST antibody as the primary antibody (71097-3; Novagen; diluted 1:10,000) at $25^{\circ} \mathrm{C}$ for $1.5 \mathrm{~h}$ and then washed 3 times with TBST buffer. Anti-mouse antibody (32430; Thermo Scientific; diluted 1:4000) conjugated to horseradish peroxidase was used as the secondary antibody. After incubation with the secondary antibody at $25^{\circ} \mathrm{C}$ for $1.5 \mathrm{~h}$, the membrane was washed 3 times. For detection, SuperSignal West Femto Maximum Sensitivity Substrate (Thermo Scientific) was used as the chemiluminescence substrate and ImageQuant LAS 4000 mini (GE Healthcare) was used for imaging.

Crystallization, data collection, and structure determination. All crystallization trials were performed by the sitting-drop vapor diffusion method. Crystals of ShD14 were obtained by mixing $0.40 \mu \mathrm{L}$ of purified protein solution $\left(6.9 \mathrm{mg} \mathrm{mL}^{-1}\right)$ with $0.40 \mu \mathrm{L}$ of reservoir solution containing $100 \mathrm{mM}$ CAPS ( $\mathrm{pH} 10.0$ ), $200 \mathrm{mM}$ $\mathrm{NaCl}$, and $20 \%(\mathrm{w} / \mathrm{v})$ PEG8000 and allowing the drop to equilibrate at $20^{\circ} \mathrm{C}$ in $96-$ well Intelli-Plates (Art Robbins). Crystals of ShHTL1 were grown by mixing $0.45 \mu \mathrm{L}$ of purified ShHTL1 protein solution $\left(15.0 \mathrm{mg} \mathrm{mL}^{-1}\right)$ with $0.45 \mu \mathrm{L}$ of reservoir solution containing $100 \mathrm{mM}$ Tris $(\mathrm{pH} 8.5), 150 \mathrm{mM} \mathrm{MgCl}_{2}$, and $23 \%$ (w/ v) PEG6000 and allowing the drop to equilibrate at $5{ }^{\circ} \mathrm{C}$. For crystallization of ShHTL4, $0.40 \mu \mathrm{L}$ of protein solution $\left(18.0 \mathrm{mg} \mathrm{mL}^{-1}\right)$ was mixed with $0.40 \mu \mathrm{L}$ of reservoir solution containing $90 \mathrm{mM}$ Tris ( $\mathrm{pH} 9.0$ ), $180 \mathrm{mM} \mathrm{MgCl}_{2}, 27 \%(\mathrm{w} / \mathrm{v}$ ) PEG4000, and $1 \mathrm{mM} \mathrm{GSH}$ and incubated at $20^{\circ} \mathrm{C}$. Crystals of ShHTL7 were grown at $20^{\circ} \mathrm{C}$ in $0.8 \mu \mathrm{L}$ droplets obtained by mixing $0.50 \mu \mathrm{L}$ of protein solution $(7.5 \mathrm{mg}$ $\mathrm{mL}^{-1}$ ) and $0.30 \mu \mathrm{L}$ of reservoir solution (90 mM Tris, $\mathrm{pH} 8.5,180 \mathrm{mM} \mathrm{MgCl}_{2}, 27 \%$ (w/v) PEG5000 and 3.5\% 1,4-dioxane).

A single crystal of ShD14 was picked up and soaked in the reservoir solution containing $20 \%(\mathrm{v} / \mathrm{v})$ ethylene glycol as a cryoprotectant, and a diffraction data set was collected on beamline AR-NW12A at the Photon Factory (Tsukuba, Japan). The diffraction data for ShHTL1 were collected on beamline PF BL-5A at the Photon Factory (Tsukuba, Japan). The diffraction data for the ShHTL4 crystal were collected by using an in-house Rigaku R-AXIS VII imaging-plate detector (Rigaku, Japan). The diffraction data for the ShHTL7 crystal were collected on beamline PF BL-17A at the Photon Factory (Tsukuba, Japan). The diffraction data were indexed integrated, and scaled using the XDS program ${ }^{47}$. The crystal structure of OsD14 (PDB code: 3VXK [https://doi.org/10.2210/pdb3VXK/pdb]) was used as a model for molecular replacement by MOLREP ${ }^{48}$. BUCCANEER ${ }^{49}$ was applied for automatic model building. Iterative refinement cycles were performed using REFMAC $^{50}$, PHENIX ${ }^{51}$, and COOT $^{52}$. Data collection and refinement statistics are presented in Table 1 . The $B$-factors of the crystal structures were calculated by Baverage $^{53}$, and the volumes of the protein cavities were calculated using the CASTp server ${ }^{54}$ with a probe radius of $1.4 \AA$. All structures were depicted by using PyMOL viewer (Version 1.8 Schrödinger, LLC). Sequence alignment was performed using CLUSTAL $\mathrm{W}^{55}$ with default parameters, and the results were displayed via ESPript $3.0^{56}$

\section{Data availability}

The atomic coordinates and structure factors of ShHTL1, ShHTL4, ShHTL7, and ShD14 have been deposited in the Protein Data Bank (PDB) under accession codes 5Z7W (https:// doi.org/10.2210/pdb5Z7W/pdb), 5Z7X, (https://doi.org/10.2210/pdb5Z7X/pdb), 5Z7Y (https://doi.org/10.2210/pdb5Z7Y/pdb), and 5Z7Z (https://doi.org/10.2210/pdb5Z7Z/pdb), 
respectively. All other data supporting the findings of this study are available within the manuscript and its supplementary files or are available from the corresponding author upon request.

Received: 27 April 2018 Accepted: 29 August 2018

Published online: 26 September 2018

\section{References}

1. Cook, C. E., Whichard, L. P., Turner, B., Wall, M. E. \& Egley, G. H. Germination of witchweed (Striga lutea Lour.): isolation and properties of a potent stimulant. Science 154, 1189-1190 (1966).

2. Berner, D. K., Kling, J. G. \& Singh, B. B. Striga research and control-a perspective from Africa. Plant Dis. 79, 652-660 (1995).

3. Ejeta, G. Integrating New Technologies for Striga Control: Towards Ending the Witch-hunt (World Scientific, Singapore, 2007).

4. Scholes, J. D. \& Press, M. C. Striga infestation of cereal crops-an unsolved problem in resource limited agriculture. Curr. Opin. Plant. Biol. 11, 180-186 (2008).

5. Akiyama, K., Matsuzaki, K. \& Hayashi, H. Plant sesquiterpenes induce hyphal branching in arbuscular mycorrhizal fungi. Nature 435, 824-827 (2005).

6. Gomez-Roldan, V. et al. Strigolactone inhibition of shoot branching. Nature 455, 189-194 (2008).

7. Umehara, M. et al. Inhibition of shoot branching by new terpenoid plant hormones. Nature 455, 195-200 (2008).

8. Flematti, G. R., Ghisalberti, E. L., Dixon, K. W. \& Trengove, R. D. A compound from smoke that promotes seed germination. Science 305, 977 (2004).

9. Flematti, G. R., Ghisalberti, E. L., Dixon, K. W. \& Trengove, R. D. Identification of alkyl substituted $2 \mathrm{H}$-Furo[2,3-c] pyran-2-ones as germination stimulants present in smoke. J. Agric. Food Chem. 57, 9475-9480 (2009).

10. Nelson, D. C. et al. Karrikins enhance light responses during germination and seedling development in Arabidopsis thaliana. Proc. Natl Acad. Sci. USA 107, 7095-7100 (2010).

11. Nelson, D. C., Flematti, G. R., Ghisalberti, E. L., Dixon, K. W. \& Smith, S. M. Regulation of seed germination and seedling growth by chemical signals from burning vegetation. Annu. Rev. Plant. Biol. 63, 107-130 (2012).

12. Chiwocha, S. D. S. et al. Karrikins: a new family of plant growth regulators in smoke. Plant Sci. 177, 252-256 (2009).

13. Conn, C. E. et al. Convergent evolution of strigolactone perception enabled host detection in parasitic plants. Science 349, 540-543 (2015).

14. Hamiaux, C. et al. DAD2 is an $\alpha / \beta$ hydrolase likely to be involved in the perception of the plant branching hormone, strigolactone. Curr. Biol. 22, 2032-2036 (2012).

15. Kagiyama, M. et al. Structures of D14 and D14L in the strigolactone and karrikin signaling pathways. Genes Cells 18, 147-160 (2013).

16. Nakamura, H. et al. Molecular mechanism of strigolactone perception by DWARF14. Nat. Commun. 4, 2613 (2013).

17. Yao, R. et al. DWARF14 is a non-canonical hormone receptor for strigolactone. Nature 536, 469-473 (2016).

18. Waters, M. T. et al. Specialisation within the DWARF14 protein family confers distinct responses to karrikins and strigolactones in Arabidopsis. Development 139, 1285-1295 (2012).

19. Bythell-Douglas, R. et al. The structure of the karrikin-insensitive protein (KAI2) in Arabidopsis thaliana. PLoS ONE 8, e54758 (2013).

20. Guo, Y., Zheng, Z., La Clair, J. J., Chory, J. \& Noel, J. P. Smoke-derived karrikin perception by the $\alpha / \beta$-hydrolase KAI2 from Arabidopsis. Proc. Natl Acad. Sci. USA 110, 8284-8289 (2013).

21. Ishikawa, S. et al. Suppression of tiller bud activity in tillering dwarf mutants of rice. Plant Cell Physiol. 46, 79-86 (2005).

22. Stirnberg, P., van De Sande, K. \& Leyser, H. M. O. MAX1 and MAX2 control shoot lateral branching in Arabidopsis. Development 129, 1131-1141 (2002).

23. Nelson, D. C. et al. F-box protein MAX2 has dual roles in karrikin and strigolactone signaling in Arabidopsis thaliana. Proc. Natl Acad. Sci. USA 108, 8897-8902 (2011).

24. Waters, M. T., Scaffidi, A., Flematti, G. R. \& Smith, S. M. Karrikins force a rethink of strigolactone mode of action. Plant Signal. Behav. 7, 969-972 (2012).

25. Morffy, N., Faure, L. \& Nelson, D. C. Smoke and hormone mirrors: action and evolution of karrikin and strigolactone signaling. Trends Genet. 32, 176-188 (2016).

26. Toh, S., Holbrook-Smith, D., Stokes, M. E., Tsuchiya, Y. \& McCourt, P. Detection of parasitic plant suicide germination compounds using a highthroughput Arabidopsis HTL/KAI2 strigolactone perception system. Chem. Biol. 21, 988-998 (2014).
27. Yao, J. et al. An allelic series at the KARRIKIN INSENSITIVE 2 locus of Arabidopsis thaliana decouples ligand hydrolysis and receptor degradation from downstream signaling. Plant J. https://doi.org/10.1111/tpj.14017 (2018).

28. Tsuchiya, Y. et al. Probing strigolactone receptors in Striga hermonthica with fluorescence. Science 349, 864-868 (2015).

29. Toh, S. et al. Structure-function analysis identifies highly sensitive strigolactone receptors in Striga. Science 350, 203-207 (2015).

30. Yao, R. et al. ShHTL7 is a non-canonical receptor for strigolactones in root parasitic weeds. Cell Res. 27, 838-841 (2017).

31. $\mathrm{Xu}$, Y. et al. Structural basis of unique ligand specificity of KAI2-like protein from parasitic weed Striga hermonthica. Sci. Rep 6, e31386 (2016).

32. Conn, C. E. \& Nelson, D. C. Evidence that KARRIKIN-INSENSITIVE2 (KAI2) receptors may perceive an unknown signal that is not karrikin or strigolactone. Front. Plant Sci. 6, 1219 (2016).

33. Zhao, L. et al. Crystal structures of two phytohormone signal-transducing $\alpha / \beta$ hydrolases: karrikin-signaling KAI2 and strigolactone-signaling DWARF14. Cell Res. 23, 436-439 (2013).

34. Flematti, G. R., Scaffidi, A., Waters, M. T. \& Smith, S. M. Stereospecificity in strigolactone biosynthesis and perception. Planta 243, 1361-1373 (2016).

35. Scaffidi, A. et al. Strigolactone hormones and their stereoisomers signal through two related receptor proteins to induce different physiological responses in Arabidopsis. Plant Physiol. 165, 1221-1232 (2014).

36. Liu, Q. et al. Striga hermonthica MAX2 restores branching but not the very low fluence response in the Arabidopsis thaliana max2 mutant. New Phytol. 202, 531-541 (2014).

37. Waters, M. T. et al. A Selaginella moellendorffii ortholog of KARRIKIN INSENSITIVE2 functions in Arabidopsis development but cannot mediate responses to karrikins or strigolactones. Plant Cell 27, 1925-1944 (2015).

38. Bythell-Douglas, R. et al. Evolution of strigolactone receptors by gradual neofunctionalization of KAI2 paralogues. BMC Biol. 15, 52 (2017).

39. Jiang, L. et al. DWARF 53 acts as a repressor of strigolactone signalling in rice. Nature 504, 401-405 (2013).

40. Zhou, F. et al. D14-SCF(D3)-dependent degradation of D53 regulates strigolactone signalling. Nature 504, 406-410 (2013),

41. Wang, L. et al. Strigolactone signaling in Arabidopsis regulates shoot development by targeting D53-like SMXL repressor proteins for ubiquitination and degradation. Plant Cell 27, 3128-3142 (2015).

42. Soundappan, I. et al. SMAX1-LIKE/D53 family members enable distinct MAX2-dependent responses to strigolactones and karrikins in Arabidopsis. Plant Cell 27, 3143-3159 (2015).

43. Stanga, J. P., Smith, S. M., Briggs, W. R. \& Nelson, D. C. SUPPRESSOR OF MORE AXILLARY GROWTH2 1 controls seed germination and seedling development in Arabidopsis. Plant Physiol. 163, 318-330 (2013).

44. Stanga, J. P., Morffy, N. \& Nelson, D. C. Functional redundancy in the control of seedling growth by the karrikin signaling pathway. Planta 243, 1397-1406 (2016).

45. Dupeux, F. et al. A thermodynamic switch modulates abscisic acid receptor sensitivity. EMBO J. 30, 4171-4184 (2011).

46. Waters, M. T., Scaffidi, A., Sun, Y. K., Flematti, G. R. \& Smith, S. M. The karrikin response system of Arabidopsis. Plant J. 79, 623-631 (2014).

47. Kabsch, W. XDS. Acta Cryst. D. 66, 125-132 (2010).

48. Vagin, A. \& Teplyakov, A. MOLREP: an automated program for molecular replacement. J. Appl. Cryst. 30, 1022-1025 (1997).

49. Cowtan, K. Fitting molecular fragments into electron density. Acta Cryst. D. 64, 83-89 (2008).

50. Murshudov, G. N. et al. REFMAC5 for the refinement of macromolecular crystal structures. Acta Cryst. D. 67, 355-367 (2011)

51. Adams, P. D. et al. PHENIX: a comprehensive Python-based system for macromolecular structure solution. Acta Cryst. D. 66, 213-221 (2010).

52. Emsley, P. \& Cowtan, K. Coot: model-building tools for molecular graphics. Acta Cryst. D. 60, 2126-2132 (2004).

53. Winn, M. D. et al. Overview of the CCP4 suite and current developments Acta Cryst. D. 67, 235-242 (2011).

54. Dundas, J. et al. CASTp: computed atas of surface topography of proteins with structural and topographical mapping of functionally annotated residues. Nucleic Acids Res. 34, 116-118 (2006).

55. Thompson, J. D., Higgins, D. G. \& Gibson, T. J. CLUSTAL W: improving the sensitivity of progressive multiple sequence alignment through sequence weighting, position specific gap penalties and weight matrix choice. Nucleic Acids Res. 22, 4673-4680 (1994).

56. Robert, X. \& Gouet, P. Deciphering key features in protein structures with the new ENDscript server. Nucleic Acids Res. 42, 320-324 (2014).

\section{Acknowledgements}

The synchrotron radiation experiments were performed on beamlines AR-NW12A, BL5A, and BL17A at the Photon Factory with the approval of the High Energy Accelerator Research Organization (Proposal No. 2015R-25). This work was supported by the 
Platform for Drug Discovery, Informatics, and Structural Life Science from the Ministry of Education, Culture, Sports, Science, and Technology of Japan (MEXT) (M.T.), the Core Research for Evolutional Science and Technology (CREST) Program of the Japan Science and Technology Agency (JST) (T.A.), and a Grant-in-Aid for Scientific Research in Innovative Areas (JP17H05835 (T.M.) and JP18H04608 (H.N.)) from the Japan Society for the Promotion of Science (JSPS) and JSPS KAKENHI (JP15H05621 (T.M.) and JP26520303 (H.N.)). We thank Ikuo Takahashi for his help with the activity measurements.

\section{Author contributions}

M.T. designed the research. Y.X. performed the biochemical experiments and collected X-ray diffraction data with T.M., S.N., A.N., Y.L. and H.N. performed the HPLC experiments. Y.X., T.M., U.O., H.I. and T.S. performed protein expression using the baculovirus-insect cell expression system. Y.X., T.M., S.N. and A.N. analyzed the data. Y.X., T.M., T.A. and M.T. wrote the paper. M.T. edited the manuscript.

\section{Additional information}

Supplementary Information accompanies this paper at https://doi.org/10.1038/s41467018-06452-2.

Competing interests: The authors declare no competing interests.
Reprints and permission information is available online at http://npg.nature.com/ reprintsandpermissions/

Publisher's note: Springer Nature remains neutral with regard to jurisdictional claims in published maps and institutional affiliations.

cc (i) Open Access This article is licensed under a Creative Commons Attribution 4.0 International License, which permits use, sharing, adaptation, distribution and reproduction in any medium or format, as long as you give appropriate credit to the original author(s) and the source, provide a link to the Creative Commons license, and indicate if changes were made. The images or other third party material in this article are included in the article's Creative Commons license, unless indicated otherwise in a credit line to the material. If material is not included in the article's Creative Commons license and your intended use is not permitted by statutory regulation or exceeds the permitted use, you will need to obtain permission directly from the copyright holder. To view a copy of this license, visit http://creativecommons.org/ licenses/by/4.0/.

(C) The Author(s) 2018 